Radial and Nonradial Pulsations as Prabes of Stellar Physics

ASP Conference Series, Vol. 259, 2002

C. Aerts, T.R. Bedding, 8 J. Christensen-Dalsgaard, eds.

\title{
Gravitational-Wave Asteroseismology as a Tool to Reveal the Equation of State of Relativistic Neutron Stars
}

\author{
Johannes Ruoff \\ Department of Physics, Aristotle University of Thessaloniki, Greece, \\ e-mail: ruoff@astro.auth.gr
}

\begin{abstract}
The equation of state (EOS) is still the big unknown in the physics of neutron stars. An accurate measurement of both the mass and the radius of a neutron star would put severe constraints on the range of possible EOSs. I discuss how the parameters of the oscillation modes of a neutron star, measured from the emitted gravitational waves, can in principle be used to infer its mass and radius, and thus reveal its EOS.
\end{abstract}

Asteroseismology is the art of looking into the interior of stellar objects through observation of their various pulsation modes. It has helped to give a profound understanding of the interior structure of our Sun, of stars and even white dwarfs. With the current gravitational wave detectors going online very soon, asteroseismology might be enriched with a completely new branch: that of gravitational-wave asteroseismology. Gravitational waves can carry information about the most violent cosmic events such as, for instance, collisions of black holes, binary neutron star mergers or supernovae. As they travel more or less undisturbed through space and time, gravitational waves carry clean information from their point of creation and could provide us with an unprecedented wealth of information about the physics involved in the most extreme places of our Universe. In particular, they might help us to reveal the still unknown equation of state of matter in the interior of neutron stars.

It is well known that neutron stars and black holes are the most compact objects of our Universe known to date. The gravitational field of these bodies is so strong that only the theory of general relativity can provide a correct description. A feature of the relativistic theory is that non-radial oscillations of these bodies give rise to the emission of gravitational waves. Carrying away energy and angular momentum, gravitational waves will in general lead to a damping of the oscillations. In the case of rapidly rotating neutron stars, the opposing effect can actually take place, i.e., the emission of gravitational waves can make certain oscillations grow. As the energy and momentum conservation laws cannot be violated, the mode must extract its energy from the rotational energy of the star, thus ultimately slowing it down. This is in particular the case for the so-called $r$ mode (Andersson \& Kokkotas, 2001). Once excited, it should not only emit copious amounts of gravitational radiation, but it also could slow down both hot young rapidly rotating neutron stars and old neutron stars spun up by accretion, thus providing an explaining mechanism to account for the observed spin distribution of pulsars, which rotate much slower than they actually could according to theory. Neutron stars possess quite a rich pulsation spectrum (Kokkotas \& Schmidt, 1999; Andersson \& Kokkotas, 2001). Most of 


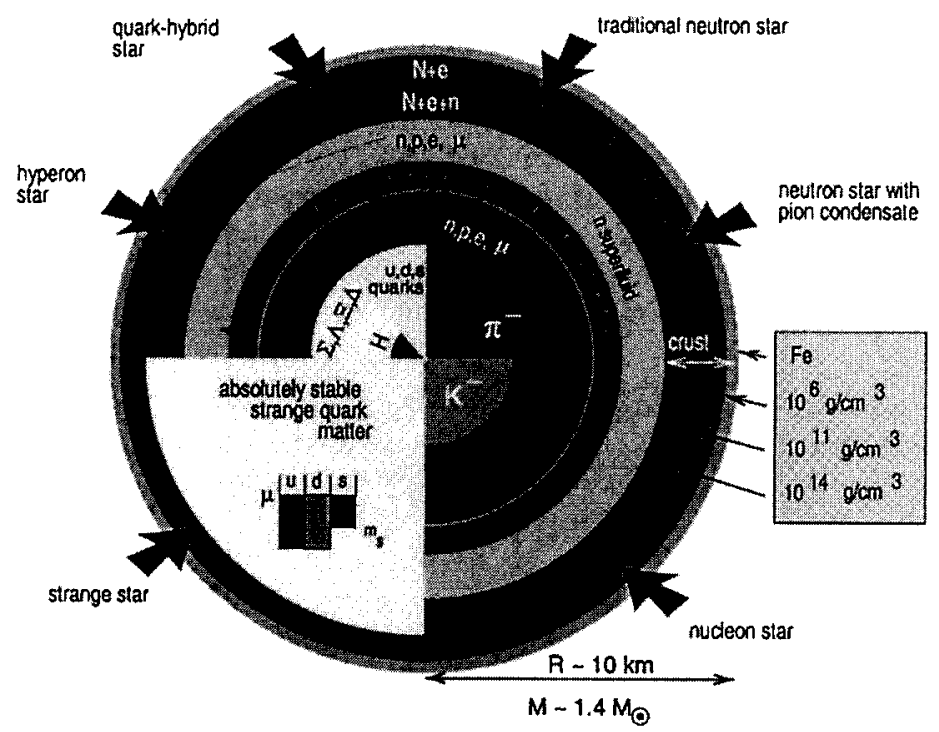

Figure 1. Competing structures and novel phases of subatomic matter predicted by theory to make their appearance in the cores $(R<$ $8 \mathrm{~km}$ ) of neutron stars. (From Weber, 2001).

the modes are also known from Newtonian stars, such as the $f$ (undamental) mode, the $p$ (ressure) and $g$ (ravity) modes, or the $t$ (orsional) modes of the crust. Rotating stars can possess additional modes such as the $r$ modes and other various rotationally induced inertial modes. A different family of modes, which is of pure relativistic origin, are the $w$ (ave) modes. They are more similar to the quasi-normal modes of black holes, which are pure spacetime oscillations. Unless the neutron star is ultra-compact, the $w$ modes decay within a few milliseconds, whereas the typical damping time of the $f$ mode lies in the range of seconds. The other modes are usually much more long-lived. Typical frequencies are 2-4 $\mathrm{kHz}$ for the $f$ mode, the $p$ and $w$ mode have frequencies above $5 \mathrm{kHz}$, whereas the $g$ and $t$ modes lie below $1 \mathrm{kHz}$.

The density inside neutron stars can easily exceed the nuclear density by up to about an order of magnitude. At such extreme densities, the physics is far from fully understood and various notions about the prevailing EOS exist, which are graphically depicted in Fig. 1 (from Weber, 2001). In the traditional view, the neutron star is made out of a $\beta$-equilibrium of neutrons, protons, electrons, muons and neutrinos. This quite oversimplified picture has been refined by more sophisticated models where various other forms of matter occur, such as hyperons or pion and kaon condensates. Another proposition is the occurrence of a more exotic form of matter such as the H-dibaryon, a doubly strange six-quark composite with zero spin and isospin. Or the baryons might start to dissolve and create a 3-flavor quark-gluon plasma containing $u, d$ and $s$ quarks. If, however, it is true that the ground state of QCD is not the most tightly bound atomic 


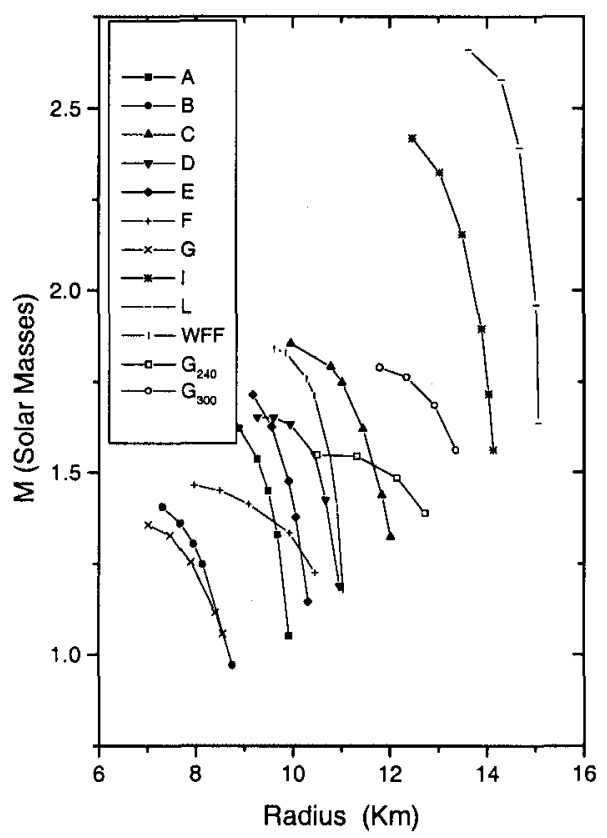

Figure 2. Mass-Radius relation of neutron stars based on various different equations of state. (From Andersson \& Kokkotas, 1998).

nucleus ${ }^{56} \mathrm{Fe}$, but instead what is called "strange (quark) matter", consisting of $u, d$ and $s$ quarks, then not only the central core but the whole neutron star would consist of strange matter, since once this phase transition occurs at some place inside the core, it would immediately induce the complete star to convert to strange matter. Consequently, such a star is called a "strange star" with quite different properties from "ordinary" neutron stars.

From Fig. 2 it is obvious that each EOS has its distinctive mass-radius relation. Hence, an accurate mass and radius determination of only a few neutron stars should be enough to exclude most of the possible EOSs. Although there exist some very precise neutron star mass measurements in binaries, the problem lies in an accurate radius determination. With conventional methods the error bars are still much too large in order to significantly restrict the range of possible EOSs. Therefore Andersson \& Kokkotas (1998) suggested to use gravitational waves of pulsating neutron stars to infer the EOS. The determination of the frequencies and damping times of the $f$, the first $p$ and the first $w$ mode could be used to infer both the mass $M$ and radius $R$ of the neutron star. This can be accomplished using a number of empirical relations which relate the mode parameters (i.e. frequency and damping time) to various combinations of $M$ and $R$. If the mode parameters can be extracted from the data of a gravitational 
wave detector, then the inversion of these laws leads to definite values of $M$ and $R$.

Of course, the success depends strongly on the accuracy of the determination of the mode parameters. A large error might result in large mass and radius uncertainties. Kokkotas et al. (2001) have investigated how accurately the mode parameters of these modes can be inferred from a noisy gravitational-wave data stream and have concluded that the generation of detectors presently under construction may well be able to observe such sources from within our galaxy. For a more exhaustive presentation of the above described method I refer to a review by Kokkotas \& Andersson (2001).

Acknowledgments. I thank Kostas D. Kokkotas for helpful comments and Fridolin Weber for the permission of using Fig. 1. The author is supported by the Marie Curie Fellowship No. HPMF-CT-1999-00364.

\section{References}

Andersson, N. \& Kokkotas, K.D. 1998, MNRAS, 299, 1059

Andersson, N. \& Kokkotas, K.D. 2001, Int. J. Mod. Phys. D, 10, 381

Kokkotas, K.D. \& Andersson, N. 2001, in Proceedings of SIGRAV XIV, Genoa 2000, (Springer-Verlag)

Kokkotas, K.D., Apostolatos, T.A., \& Andersson, N. 2001, MNRAS, 320, 307

Kokkotas, K.D. \& Schmidt, B.G. 1999, http://www.livingreviews.org/Articles/ Volume2/1999-2kokkotas/index.html

Weber, F., 2001, J. Phys., G27, 465

\section{Discussion}

I. Roxburgh : Wouldn't you expect the neutron stars to have a solid crust, and how would this effect your calculations?

J. Ruoff : Yes, certainly the crust would have some effects, in particular for the $f$ and $p$ modes. The $w$ modes, however, should not be affected very much.

I. Roxburgh : You mention rotation but these stars also have very strong magnetic fields. Would these be important?

J. Ruoff : This depends on the strength of the magnetic fields. For magnetars which can have field strengths up to $10^{16}$ Gauss, the influence is not negligible and the magnetic field has to be included in the calculation of the stellar models and their associated modes. Actually, there is much work in progress including more physics such as a solid crust, magnetic fields, rapid rotation, superfluid cores etc. 